\title{
Reparable Superhydrophobic Surface with Hidden Reactivity, Its Photofunctionalization and Photopatterning
}

\author{
Xin Du,* Min Wang, Alexander Welle, Farid Behboodi-Sadabad, YuLi Wang, \\ Pavel A. Levkin,* and Zhongze Gu*
}

Superhydrophobic $(\mathrm{SH})$ surfaces have held great promise during the last decade because of their diverse potential applications as self-cleaning surfaces, in microfluidics, microarrays, etc. Recently, reactive $\mathrm{SH}$ surfaces combining their liquid-repellency with the possibility for convenient postfunctionalization and patterning were introduced. However, current concepts for making reactive $\mathrm{SH}$ materials have very limited selection of "hydrophobic as well as reactive" groups. Here a novel reparable $\mathrm{SH}$ surface with hidden reactivity (SuSHiR) based on photodynamic properties of disulfides is reported. Reactive hydrophobic, $\mathrm{SH}$, as well as superhydrophilic surfaces are fabricated using this method. The method allows the use of diverse end functionalities (reactive or nonreactive, hydrophobic, or hydrophilic), while keeping the same reactive disulfides "hidden" in the middle of the functional surface-grafted chains. The photoreactivity of the disulfide bond allows for the functionalization and patterning of various molecules through UV irradiation. The obtained SuSHiR exhibits better stability compared to conventional reactive $\mathrm{SH}$ surfaces, and the reversibility of the disulfide-disulfide exchange reaction enables reversible patterning as well as photorestoration of damaged functionalities.

\section{Introduction}

Liquid-repellent and superhydrophobic ( $\mathrm{SH}$ ) surfaces are promising materials both for industrial and academic fields, with applications ranging from self-cleaning coatings, oil-water separation membranes, water transfer systems to biotechnology, microfluidics, and microarrays. ${ }^{[1-9]}$ For the application

Dr. X. Du, M. Wang, Y. L. Wang, Prof. Z. Z. Gu

State Key Laboratory of Bioelectronics

Southeast University

210096 Nanjing, China

E-mail: du.xin@seu.edu.cn; gu@seu.edu.cn

Dr. A. Welle

Institute of Functional Interfaces

Karlsruhe Institute of Technology

76131 Karlsruhe, Germany

Dr. A. Welle

Karlsruhe Nano Micro Facility

Karlsruhe Institute of Technology

76131 Karlsruhe, Germany of SH surfaces, however, further functionalization and patterning of wettabilities or desired molecules are often required, which is difficult to achieve due to the inertness and low reactivity of most of $\mathrm{SH}$ surfaces. ${ }^{[10-14]}$ Recently, reactive SH surfaces combining their liquid-repellency with the possibility for further functionalization were introduced. ${ }^{[15,16]}$ Such surfaces exhibit liquid-repellent properties and show reactivity to specific functional groups, allowing for simple postfunctionalization and patterning. Apart from reactive $\mathrm{SH}$ surfaces, reactive slippery surfaces have been also reported. ${ }^{[17]}$ The principle for the reactive $\mathrm{SH}$ surface is shown in Figure 1a. The top of the hydrophobic surface is functionalized with reactive functional groups to endow the substrate reactivity, while the liquid-repellent property being provided either by the reactive groups themselves (Figure S1a, Supporting Information) $)^{[15,16]}$ or by the bulk polymer where the reactive groups are distributed (Figure S1b, Supporting Information). ${ }^{[17]}$ By the combination of reactive moieties with hydrophobic or oleophobic moieties, reactive surfaces with liquid-repellent properties can be obtained.

Despite the progress in this field, existing methods for creating reactive $\mathrm{SH}$ surfaces possess limitations. For example, if both the hydrophobicity and reactivity are provided by the same

\author{
F. Behboodi-Sadabad, Dr. P. A. Levkin \\ Institute of Toxicology and Genetics \\ Karlsruhe Institute of Technology (KIT) \\ 76344 Eggenstein-Leopoldshafen, Germany \\ E-mail: pavel.levkin@kit.edu \\ Y. L. Wang \\ Jiangsu Key Laboratory of Oral Diseases \\ Nanjing Medical University \\ 210096 Nanjing, China \\ F. Behboodi-Sadabad, Dr. P. A. Levkin \\ Institute of Organic Chemistry \\ Karlsruhe Institute of Technology (KIT) \\ 76131 Karlsruhe, Germany
}


(a)

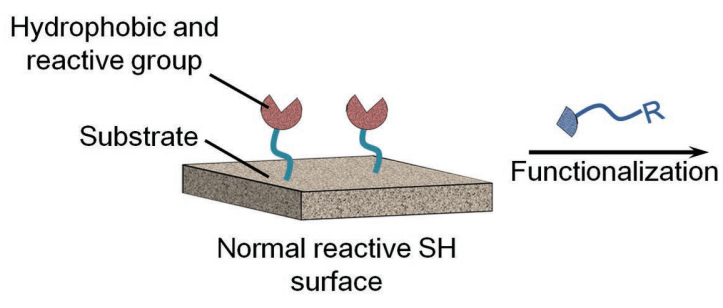

(b)

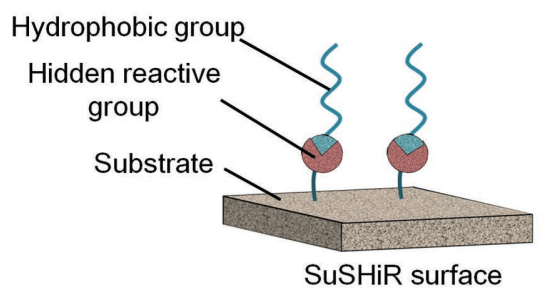

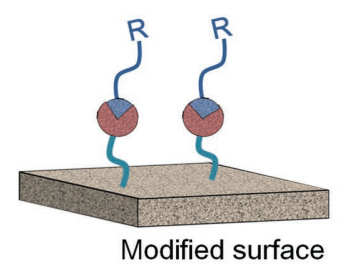

Modified surface

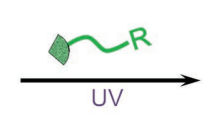

UV

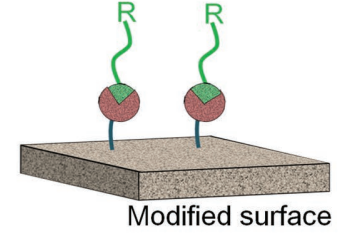

(c)

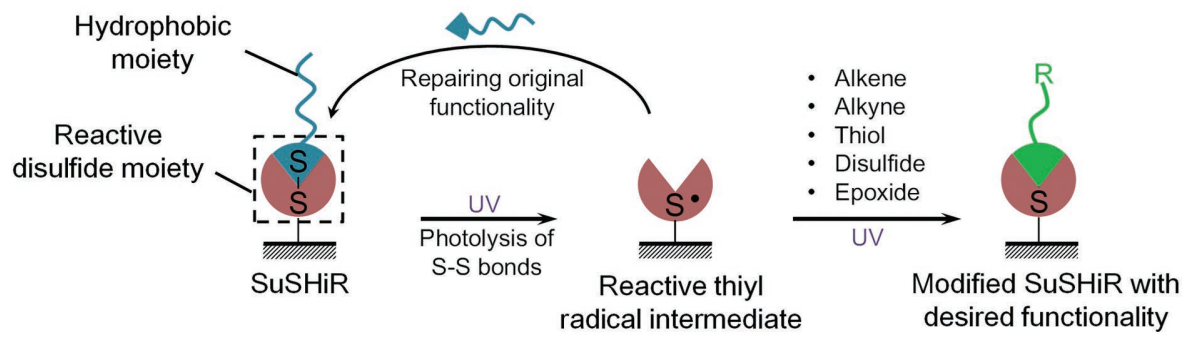

Figure 1. Schematic representation of the design of reactive superhydrophobic and liquid-repellent surfaces. a) Conventional reactive superhydrophobic surface uses reactive functional groups located at the end of hydrophobic moieties. b) On the superhydrophobic surface with hidden reactivity (SuSHiR) reactive disulfide moieties are "hidden" in the middle of the hydrophobic chains, which separates hydrophobic from the reactive surface properties. During the modification, the peripheral hydrophobic chains are substituted by a new functionality. c) Schematic showing possible chemical transformations using the SuSHiR concept. The location of the disulfide bond enables the use of different hydrophobic moieties for achieving the superhydrophobicity. UV irradiation cleaves the disulfides to thiyl radicals, causing the removal of the hydrophobic chains, while the thiyl radicals can react with various molecules, resulting either in the secondary modification or in the repair of the original functionality.

functional group, ${ }^{[15,16]}$ these moieties have to be both hydrophobic and reactive (Figure S1a, Supporting Information). This limits the selection of suitable functional groups. Currently, only two functional groups have been reported: azlactones, reactive to nucleophiles, ${ }^{[15,18]}$ and alkenes that can react with thiols or disulfides under UV irradiation. ${ }^{[16]}$ In another example, ${ }^{[17]}$ reactivity was provided by hydrophilic amines (Figure S1b, Supporting Information). To enhance the liquid-repellency, polydimethylsiloxane had to be introduced to minimize the negative effect of the amine groups. These problems limit possible design and applications of reactive $\mathrm{SH}$ and slippery interfaces.

The recently reported reversible surface functionalization strategies ${ }^{[19-21]}$ gave us an inspiration for a new kind of reactive surface, where the reactive disulfide groups are hidden by hydrophobic chains (Figure 1b,c) and the postmodification is achieved by substituting the hydrophobic chains with other molecules via the photodisulfide exchange reaction (Figure 1b,c). By using this strategy, the surface functionality (e.g., hydrophobicity or hydrophilicity) becomes disconnected from the reactivity of the disulfides hidden in the middle of the molecular layer, extending possibilities for the design of reactive interfaces with special surface properties, such as superhydrophobicity.

To realize this concept of a SH surface with hidden reactivity (SuSHiR) we employed dynamic disulfide exchange reaction
(Figure 1c). ${ }^{[19]}$ The obtained SuSHiR is more stable compared to conventional reactive $\mathrm{SH}$ surfaces due to the fact that the reactive groups are hidden under the hydrophobic ones, and enable fast UV-induced restoration of the surface functionality utilizing the photodynamic disulfide exchange reaction. The generated surfaces are reactive to alkenes, thiols, disulfides, and epoxides under UV irradiation, allowing facile postmodifications and patterning.

\section{Results and Discussions}

The disulfide substrate was prepared by esterification of hydroxy functionalized porous poly(2-hydroxyethyl methacrylate-coethylene dimethacrylate) (HEMA-EDMA) as well as a flat glass substrate modified with hydroxyethyl groups (see supporting information) with bis(carboxyethyl) disulfide (CED). ${ }^{[19]}$ The produced surface was functionalized to create SuSHiR using three different strategies: a) disulfide exchange between 2-hydroxyethyl disulfide (HED) and the CED surface, followed by the treatment with $1 H, 1 H, 2 H, 2 H$-perfluorooctyldimethylchlorosilane (FOS) to generate a fluorinated FOS-surface (Figure 2a); b) photoinduced thiol-disulfide exchange reaction between $1 \mathrm{H}, 1 \mathrm{H}, 2 \mathrm{H}, 2 \mathrm{H}$ perfluorodecanethiol (FDT) and CED surface (Figure 2b, FDT surface); c) photoinduced disulfide-disulfide exchange between 


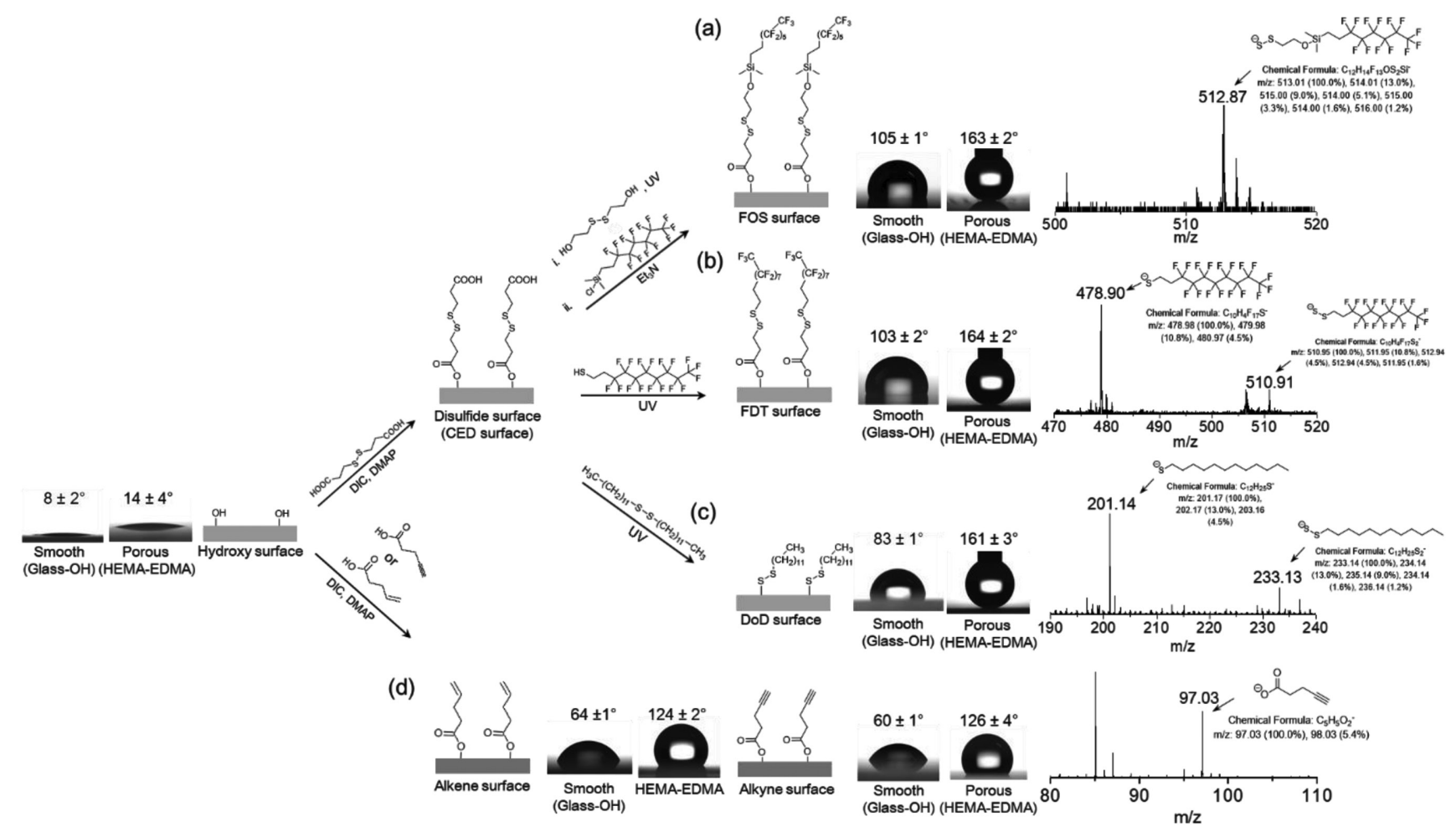

Figure 2. Formation of superhydrophobic surfaces with hidden reactivity (SUSHiR) and their ToF-SIMS analysis. a) FOS surface, formed by treating hydroxyl disulfide surface with $1 H, 1 H, 2 H, 2 H$-perfluorooctyldimethylchlorosilane. b) FDT surface, formed by UV-induced thiol-disulfide exchange on the CED disulfide surface. c) DoD surface, formed by UV-induced disulfide-disulfide exchange on the CED disulfide surface. d) Alkene and alkyne surfaces, formed by the corresponding esterification reaction on the porous HEMA-EDMA surface.

didodecyl disulfide (DoD) ${ }^{[29]}$ and CED surface leading to a liquid-repellent dodecyl-modified surface (Figure 2c, DoD surface). Alkene and alkyne modified surfaces, formed by esterification of surface hydroxy groups with 4-pentenoic or 4-pentynoic acid, respectively, were also fabricated for comparisons (conventional reactive $\mathrm{SH}$ surface, Figure $2 \mathrm{~d}$ ).

No difference could be seen in the scanning electron microscopy (SEM) images of the bare HEMA-EDMA and disulfide modified surfaces (Figure S2, Supporting Information). The presence of hydrophobic groups on all SuSHiRs was confirmed by the change of the apparent water contact angle (WCA; from $\approx 8^{\circ}$ to $>60^{\circ}$ on glass, and $\approx 14^{\circ}$ to $>124^{\circ}$ on HEMA-EDMA) before and after modification (Figure 2 and Table 1). Time-offlight secondary ion mass spectrometry (ToF-SIMS) analysis on the surfaces showed the presence of $\mathrm{C}_{12} \mathrm{H}_{14} \mathrm{~F}_{13} \mathrm{OS}_{2} \mathrm{Si}^{-}$(Figure 2a), $\mathrm{C}_{10} \mathrm{H}_{4} \mathrm{~F}_{17} \mathrm{~S}_{2}^{-}$(Figure 2b), $\mathrm{C}_{12} \mathrm{H}_{25} \mathrm{~S}_{2}^{-}$(Figure 2c), and $\mathrm{C}_{5} \mathrm{H}_{5} \mathrm{O}_{2}^{-}$ (Figure 2d) anions on FOS, FDT, DoD, and alkyne surfaces, respectively, therefore confirming the successful attachment of the corresponding hydrophobic chains to these surfaces. The presence of disulfide, alkene, and alkyne functionalities were also confirmed by Raman spectra (Figure 3a), where characteristic peaks of disulfides $\left(\approx 507 \mathrm{~cm}^{-1}\right), \mathrm{C}-\mathrm{C}$ double bond $\left(\approx 1680 \mathrm{~cm}^{-1}\right)$, and triple bond $\left(\approx 2120 \mathrm{~cm}^{-1}\right)$ could be observed, respectively.

Apparent WCA on porous HEMA-EDMA surfaces functionalized with alkene and alkyne groups (conventional reactive hydrophobic surface) were $124^{\circ} \pm 2^{\circ}$ and $126^{\circ} \pm 4^{\circ}$ (Figure 2), respectively, and the surfaces exhibit very high WCA hysterisis (as confirmed by the large difference between advancing WCA and receding WCA, Table 1), demonstrating the difficulty in creating $\mathrm{SH}$ surfaces using reactive peripheral functional groups. FOS, FDT, and DoD SuSHiRs, on the other hand, exhibited much higher apparent WCAs: $163^{\circ} \pm 2^{\circ}, 164^{\circ} \pm 2^{\circ}, 161^{\circ} \pm 3^{\circ}$, respectively. All three types of SuSHiRs are $\mathrm{SH}$ (Video S1, Supporting Information), as confirmed by the high apparent WCA and low WCA hysterisis (Table 1), while we could not achieve superhydrophobicity on the alkene and alkyne modified

Table 1. WCA of the surfaces before and after modification.

\begin{tabular}{|c|c|c|c|c|c|c|c|}
\hline Substrate & WCA type & Original & FOS & FDT & DoD & Alkene & Alkyne \\
\hline Glass & Apparent & $8^{\circ} \pm 2^{\circ}$ & $105^{\circ} \pm 1^{\circ}$ & $103^{\circ} \pm 2^{\circ}$ & $83^{\circ} \pm 1^{\circ}$ & $64^{\circ} \pm 1^{\circ}$ & $60^{\circ} \pm 1^{\circ}$ \\
\hline \multirow[t]{3}{*}{ HEMA-EDMA } & Apparent & $14^{\circ} \pm 4^{\circ}$ & $163^{\circ} \pm 2^{\circ}$ & $164^{\circ} \pm 2^{\circ}$ & $161^{\circ} \pm 3^{\circ}$ & $124^{\circ} \pm 2^{\circ}$ & $126^{\circ} \pm 4^{\circ}$ \\
\hline & Advancing & $25^{\circ} \pm 2^{\circ}$ & $171^{\circ} \pm 4^{\circ}$ & $169^{\circ} \pm 2^{\circ}$ & $164^{\circ} \pm 1^{\circ}$ & $132^{\circ} \pm 3^{\circ}$ & $135^{\circ} \pm 5^{\circ}$ \\
\hline & Receding & $0^{\circ}$ & $158^{\circ} \pm 2^{\circ}$ & $153^{\circ} \pm 3^{\circ}$ & $148^{\circ} \pm 2^{\circ}$ & $0^{\circ}$ & $0^{\circ}$ \\
\hline
\end{tabular}


(a)

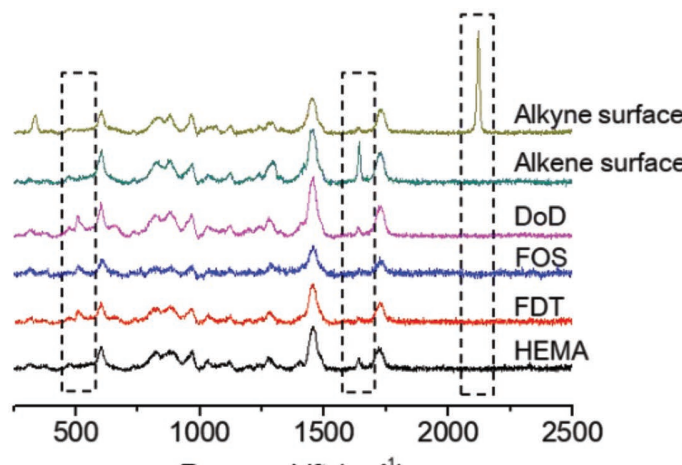

Raman shift $\left(\mathrm{cm}^{-1}\right)$

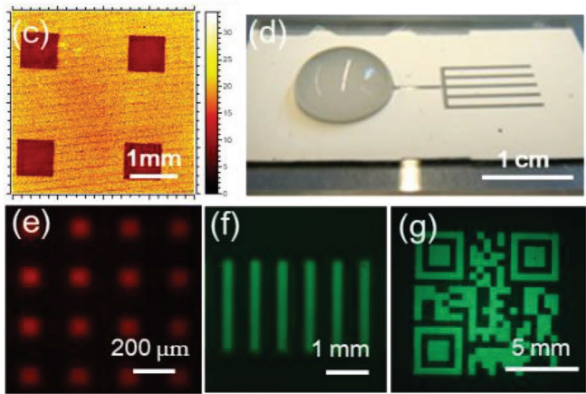

(b)

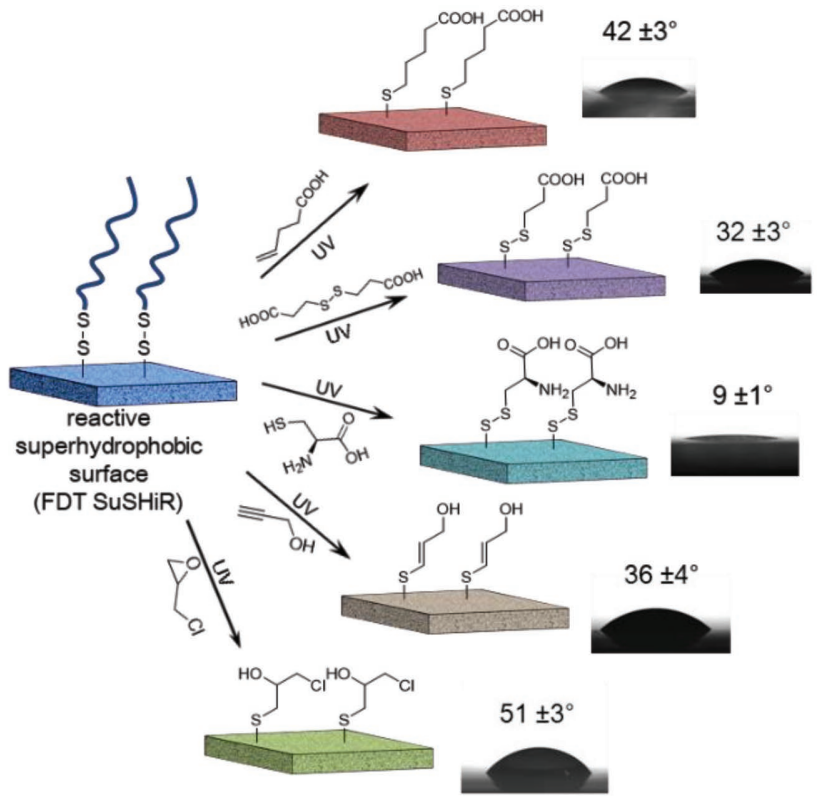

Figure 3. Functionalization and patterning of the superhydrophobic surfaces with hidden reactivity (SuSHiR). a) Raman spectra of HEMA-EDMA (HEMA), FDT, FOS, DoD and alkyne surface, and FDT surface modified with 4-pentenoic acid (alkene surface). b) Examples of possible UV-induced functionalizations of SuSHiR (FDT) with various hydrophilic molecules. Apparent WCAs before and after modification are shown. c) ToF-SIMS scan showing distribution of HS-S- (disulfide) fragment on the FDT surface patterned with 4-pentenoic acid. d) Superhydrophobic-superhydrophilic microfluidic pattern on a DRSS substrate, formed by modifying DoD surface with CED under a photomask. e-g) Different patterns formed by modifying FDT surface with rhodamine-SH, FITC-yne, and FITC-SH, respectively.

HEMA-EDMA surfaces. The SuSHiR strategy allows the complete separation of hydrophobic groups and reactive groups on the water-repellent surfaces, enabling the use of various nonreactive or poorly reactive hydrophobic groups to generate reactive water-repellent surfaces with different functionalities.

In order to demonstrate this flexibility, we prepared reactive hydrophobic surfaces by modifying porous HEMA-EDMA substrates with phenyl (Figure S3a, Supporting Information), thiophenyl (Figure S3b, Supporting Information), or Iniferter group (often used in living polymerization; Figure S3c, Supporting Information) as the hydrophobic moieties. These examples show the possibility to introduce further functionalities to SuSHiR, for example, to achieve surface conductivity or perform surface modification by grafting polymerization, while keeping the inherent "hidden" reactivity of the surface. This is a significant advantage compared with conventional reactive hydrophobic surfaces, ${ }^{[15,16]}$ because the selection of reactive and simultaneously hydrophobic functional groups is very limited.

The disulfide bonds not only offer wide selections for the hydrophobic moieties, but also endow the surfaces with the reactivity toward various commonly used functional groups. As shown in Figure 1c, UV irradiation of the disulfide surface dissociates the disulfide bond to form thiyl radicals, ${ }^{[22-24]}$ which can react with various molecules such as alkenes, alkynes, ${ }^{[16,25]}$ thiols, ${ }^{[26]}$ disulfides, ${ }^{[19]}$ and epoxides. ${ }^{[27]}$ To confirm this, we modified the FDT disulfide SH surface with several hydrophilic molecules (Figure 2b) under UV (5 min, $10 \mathrm{~mW} \mathrm{~cm}{ }^{-2}$ at $365 \mathrm{~nm}$ ). After modification the initially hydrophobic
(WCA, $161^{\circ} \pm 3^{\circ}$ ) disulfide surface became hydrophilic (WCA $9^{\circ}-51^{\circ}$ ), indicating the attachment of hydrophilic moieties. The Raman spectra of these modified surfaces are shown in Figure S4 (Supporting Information). The disulfide surface modified with 4-pentenoic acid (Figure 2a) shows the disappearance of the disulfide peak $\left(\approx 507 \mathrm{~cm}^{-1}\right)$ after modification. ${ }^{[16]}$ The consumption of disulfide bond on the 4-pentenoic acid modified disulfide surface was also confirmed by ToF-SIMS measurement (Figure 3c and Figure S5, Supporting Information) on a patterned FDT surface, that clear patterns were observed at $m / z=31.98,63.95$, and 64.96, referring to the fragments of sulfur $\left(\mathrm{S}^{-}\right)$, disulfide $\left(\mathrm{S}_{2}^{-}\right)$, and $\mathrm{HS}^{-} \mathrm{S}^{-}$anions, respectively. A drop of the intensity of these anions could be observed on the irradiated area, indicating the reaction of the disulfides with 4-pentenoic acid. However, for the FDT surface modified with hydrophilic thiols and disulfides (Figure 3b), the disulfide peak was still clearly visible, although the surfaces became hydrophilic after the modification (Figure S4, Supporting Information). This confirms the surface modifications by reversible thiol-disulfide or disulfide-disulfide exchange reactions proceeding without the loss of disulfides. ${ }^{[19,28]}$

To demonstrate the ability to control surface functionalization on SuSHiR spatially, we created SH-superhydrophilic patterns by modifying the DoD surface with hydrophilic HED (Figure 3d). To make the pattern visible under fluorescence microscope, three different reactive dyes, FITC-SH, ${ }^{[30]}$ Rhodamine-SH, and FITC-yne (Figure S6a, Supporting Information) were synthesized and patterned on the FDT SuSHiR. Different 
(a)

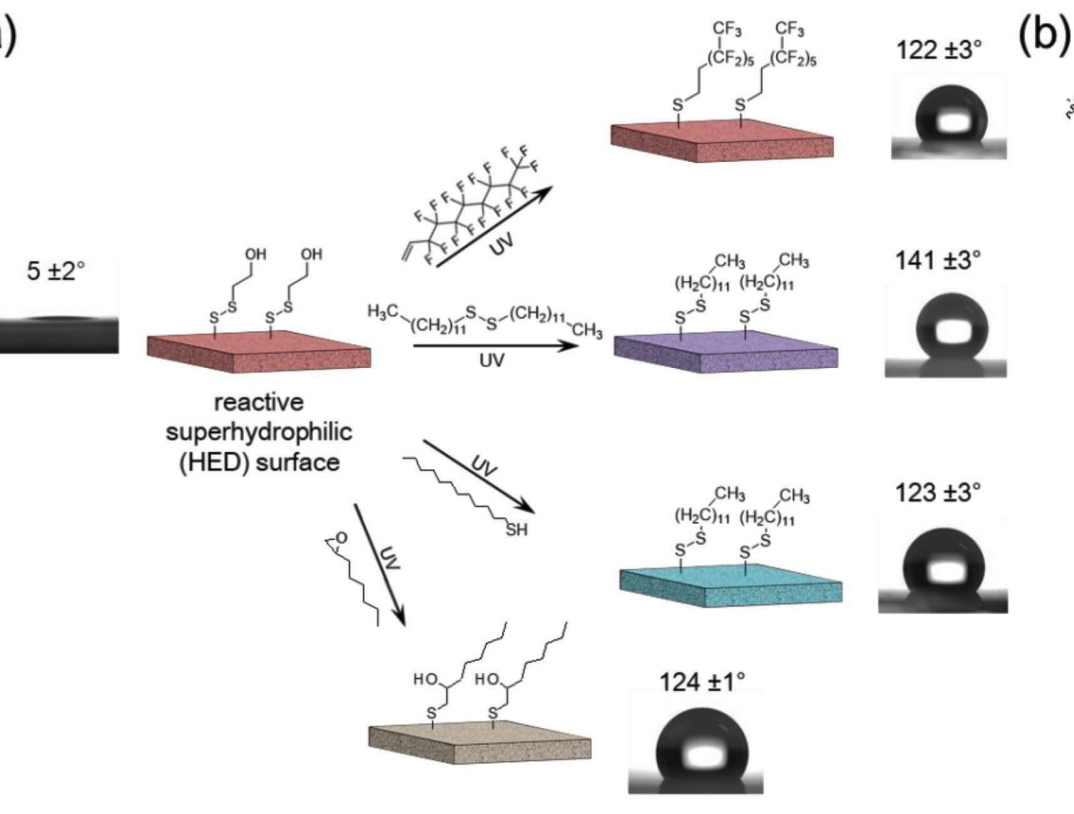

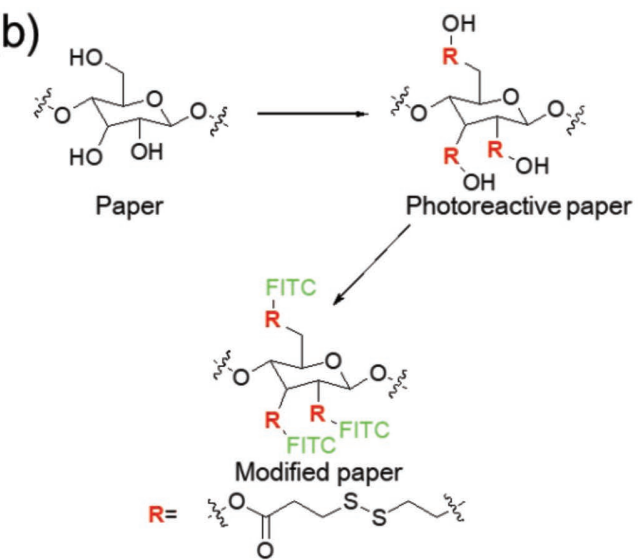
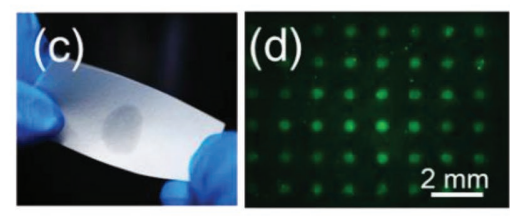

Figure 4. Disulfide-based photoreactive superhydrophilic surface and its application on paper. a) Disulfide-based photoreactive superhydrophilic surface shows a WCA of $5^{\circ} \pm 2^{\circ}$, and can be modified with different molecules inculuding alkenes, disulfides, thiols, and epoxides. b-d) Application of the disulfide-based photoreactive superhydrophilic surface onto filter paper leads to a photoreactive paper that exhibits similar wettability and mechanical performance as normal filter paper, but can be further functionalized and patterned. b) Schematic illustrating the principle of photoreactive paper. c) Photo of the obtained photoreactive paper wetted with a water drop. d) Fluorescence pattern image obtained by patterning FITC-SH onto photoreactive paper.

patterns, including simple arrays or lines (Figure $3 \mathrm{e}, \mathrm{f}$ and Figure S6b, Supporting Information) and more complex geometries (Figure $3 \mathrm{~g}$ ) could be obtained.

The SuSHiR strategy can also be applied to create a superhydrophilic surface with hidden reactivity. Thus, a reactive superhydrophilic (WCA $5^{\circ} \pm 2^{\circ}$ ) disulfide surface could be obtained by modifying the 2-carboxyethyl disulfide surface (CED surface) with HED surface (Figure 4a). This hydrophilic disulfide surface can potentially lead to the photoreactive paper, fabrics, hydrogels, or other materials that can maintain their highly hydrophilic nature, while being directly functionalizable via photochemical reactions. As an example, we modified filter paper with CED to generate reactive paper (Figure 4b) with similar wettability and mechanical performance as the original filter paper (Figure 4c). The resulting paper can be functionalized and patterned using UV irradiation (Figure 4d) and might find paper-based biological applications. The wettability-independent reactivity and photoinduced disulfidedisulfide exchange ${ }^{[19]}$ makes it possible to erase patterns, to restore functionality or achieve reusability of functional materials (Figure S7, Supporting Information).

The stability of the liquid-repellency is important for all reactive liquid-repellent interfaces, since reactivity is typically accompanied by low stability. We therefore investigated the stability of FOS, FDT, and DoD SuSHiRs as well as the alkene surface under prolonged UV irradiation $\left(10 \mathrm{~mW} \mathrm{~cm}^{-2}\right.$ at $\left.365 \mathrm{~nm}\right)$ or by storing the surfaces at $50{ }^{\circ} \mathrm{C}$ for $30 \mathrm{~d}$, and WCAs were measured at different time points. Figure 5 a shows the WCA
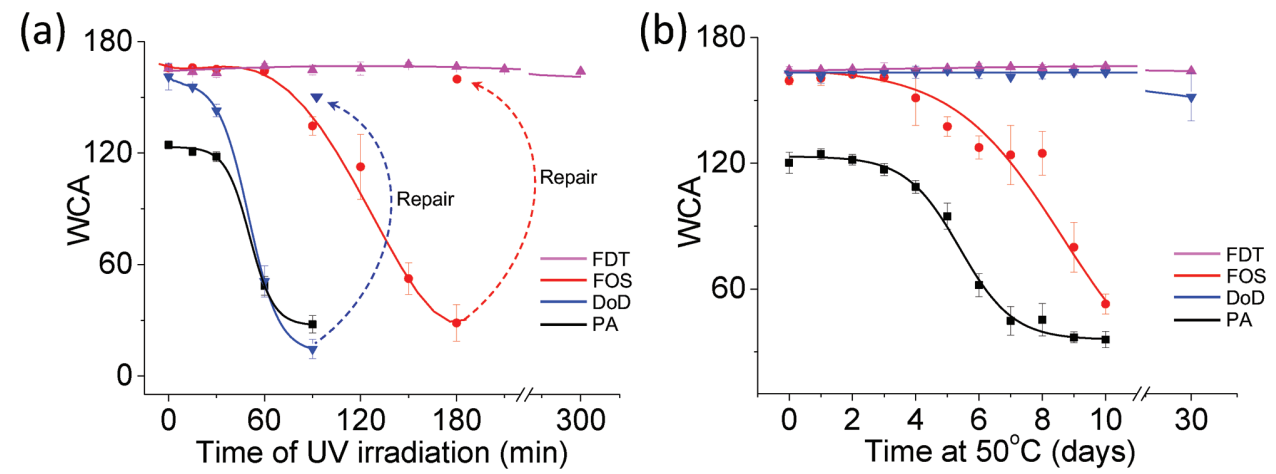

Figure 5. Stability test of different SuSHiR under UV irradiation and at $50^{\circ} \mathrm{C}$. a) Time-dependent apparent WCA change on FDT, FOS, DoD, and the PA surface under UV irradiation $\left(10 \mathrm{~mW} \mathrm{~cm}{ }^{-2}\right.$ at $\left.365 \mathrm{~nm}\right)$. The reduced hydrophobicity can be restored by 5 min UV irradiation in the presence of corresponding disulfides. b) Time-dependent WCA change on FDT, FOS, DoD, and PA surfaces during storage at $50{ }^{\circ} \mathrm{C}$. 
change of the surfaces under UV irradiation; a drop of WCA is observed on the DoD and alkene surfaces from $161^{\circ} \pm 3^{\circ}$ and $124^{\circ} \pm 2^{\circ}$ to $22^{\circ} \pm 3^{\circ}$ and $39^{\circ} \pm 2^{\circ}$, respectively. On the contrary, the perfluorinated surfaces (FOS and FDT surface) exhibited much higher stability, probably because of the higher stability of $\mathrm{C}-\mathrm{F}$ bonds to photooxidation. The WCA on the FDT surface decreased by only $\approx 3^{\circ}$ after $5 \mathrm{~h}$ of UV irradiation. WCA of FOS surface gradually decreased from $163^{\circ} \pm 2^{\circ}$ down to $28^{\circ} \pm 3^{\circ}$ after $3 \mathrm{~h}$ UV irradiation. This is probably due to the low stability of dimethylsilyl group ( $\left.-\mathrm{Si}\left(\mathrm{CH}_{3}\right)_{2}-\right)$. Due to the reversibility of the disulfide exchange reaction, it is possible to recover the hydrophobicity of the surfaces. For example, UV irradiation of the photooxidized DoD surface for $5 \mathrm{~min}$ in the presence of DoD disulfide recovers the hydrophobicity of the original surface and increases the WCA from $22^{\circ}$ to $148 \pm 2^{\circ}$ (Figure 5a). Similarly, the disulfide exchange reaction (see Figure 2a) performed on the photooxidized FOS surface almost completely recovers the original WCA of $156^{\circ} \pm 4^{\circ}$ (Figure 5a). Since after the repair step the disulfide bonds are still present on the surface, the repair step can be repeated multiple times. As an illustration, we irradiated the repaired DoD surface with UV for $1.5 \mathrm{~h}$ to degrade the superhydrophobicity by oxidation of the superficial hydrophobic groups, followed by repairing the hydrophobicity using the UV-induced disulfide exchange reaction (Figure S8a, Supporting Information). The damage-repair cycle was repeated four times and the apparent WCAs were measured after each step. As shown in Figure S8b (Supporting Information), after four damage-repair cycles, the DoD SuSHiR is still highly hydrophobic. This demonstrates the unique UVinduced reparability of SuSHiRs and enables restoration of damaged hydrophobicity (or other functionalities) by this facile method, which not only prolongs the lifetime of these functional surfaces but will also lead to various novel applications requiring the reparable and reusable functional interfaces.

Stability of the surfaces at room temperature did not show any decrease of WCAs even after one month of storage. Similar experiment under $50^{\circ} \mathrm{C}$ is shown in Figure 5b. Similar to its performance under UV, the hydrophobic alkene surface started to lose its hydrophobicity after $4 \mathrm{~d}$ at $50^{\circ} \mathrm{C}$, and the WCA dropped from $122^{\circ} \pm 3^{\circ}$ to $32^{\circ} \pm 2^{\circ}$ in 10 d. FOS surface exhibited similar tendency, becoming hydrophilic (WCA $57^{\circ} \pm 3^{\circ}$ ) after $10 \mathrm{~d}$ at $50{ }^{\circ} \mathrm{C}$, probably due to the instability of the dimethylsilyl group. For FDT and DoD surface, however, WCA of the surfaces only decreased less than $10^{\circ}$ after $30 \mathrm{~d}$ at $50^{\circ} \mathrm{C}$, demonstrating excellent stability of these surfaces.

\section{Conclusions}

In conclusion, we developed a new strategy for creating reparable superhydrophobic surface with hidden reactivity (SuSHiR) using photodynamic properties of disulfides. We fabricated reactive hydrophobic, $\mathrm{SH}$ as well as superhydrophilic surfaces, using this method. Unlike conventional reactive liquid-repellent surfaces reported before, our method allows the use of diverse end functionalities (reactive or nonreactive, hydrophobic, or hydrophilic), while keeping the same reactive disulfides "hidden" in the middle of the functional surface-grafted chains. This concept decouples the macroscopic functionality of the surface (for example, superhydrophobicity or hydrophilicity) from the photodynamic reactivity of the surface, making such surfaces and their applications more versatile. The photoreactivity of the disulfide bond allows for the functionalization and patterning of various molecules through UV irradiation. The reversibility of the disulfide-disulfide exchange reaction enables reversible patterning as well as photorestoration of damaged functionalities. We anticipate that the developed method will generate a series of novel reparable and reactive dynamic liquid-repellent or other functional surfaces, which will inevitably lead to various novel applications.

\section{Supporting Information}

Supporting Information is available from the Wiley Online Library or from the author.

\section{Acknowledgements}

The research was supported by the Fundamental Research Funding from Southeast University (1107037114) and Jiangsu province (BK20170662). P.A.L. thanks the support from European Research Council starting grant (ERC-2013-StG 337077-DropCellArray). Z.Z.G. thanks the funding from National Key R\&D Program of China (No. 2017YFA0700500) and the National Science Foundation of China (No. 21327902).

\section{Conflict of Interest}

The authors declare no conflict of interest.

\section{Keywords}

disulfide exchange, patterning, photochemistry, reactive superhydrophobic surfaces

[1] X. M. Li, D. Reinhoudt, M. Crego-Calama, Chem. Soc. Rev. 2007, 36, 1350.

[2] T. Sun, L. Feng, X. Gao, L. Jiang, Acc. Chem. Res. 2005, 38, 644.

[3] M. Ma, R. M. Hill, G. C. Rutledge, Nat. Mater. 2003, 2, 457.

[4] P. A. Levkin, F. Svec, J. M. J. Frechet, Adv. Funct. Mater. 2009, 19, 1993.

[5] E. Ueda, P. A. Levkin, Adv. Mater. 2013, 25, 1234.

[6] S. Xing, R. S. Harake, T. Pan, Lab Chip 2011, 11, 3642.

[7] A. Tropmann, L. Tanguy, P. Koltay, R. Zengerle, L. Riegger, Langmuir 2012, 28, 8292

[8] F. Mumm, A. T. J. Van Helvoort, P. Sikorski, ACS Nano 2009, 3, 2647.

[9] H. Bai, L. Wang, J. Ju, R. Sun, Y. Zheng, L. Jiang, Adv. Mater. 2014, 26, 5025.

[10] H. S. Lim, J. T. Han, D. Kwak, M. Jin, K. Cho, J. Am. Chem. Soc. 2006, 128, 14458.

[11] D. Zahner, J. Abagat, F. Svec, J. M. J. Fréchet, P. A. Levkin, Adv. Mater. 2011, 23, 3030

[12] H. Pazokian, A. Selimis, J. Barzin, S. Jelvani, M. Mollabashi, C. Fotakis, E. Stratakis, J. Micromech. Microeng. 2012, 22, 35001. 
[13] L. Xu, W. Chen, A. Mulchandani, Y. Yan, Angew. Chem., Int. Ed. 2005, 44, 6009.

[14] E. Bormashenko, S. Balter, A. Malkin, D. Aurbach, Macromol. Mater. Eng. 2014, 299, 27.

[15] U. Manna, A. H. Broderick, D. M. Lynn, Adv. Mater. 2012, 24, 4291.

[16] J. Li, L. Li, X. Du, W. Feng, A. Welle, O. Trapp, Nano Lett. 2015, 15, 675.

[17] P. Liu, H. Zhang, W. He, H. Li, J. Jiang, M. Liu, H. Sun, M. He, J. Cui, L. Jiang, X. Yao, ACS Nano 2017, 11, 2248.

[18] M. C. D. Carter, D. M. Lynn, Chem. Mater. 2016, 28, 5063.

[19] X. Du, J. Li, A. Welle, L. Li, W. Feng, P. A. Levkin, Adv. Mater. 2015, 27, 4997.

[20] S. Arumugam, V. V. Popik, J. Am. Chem. Soc. 2012, 134, 8408.

[21] N. R. Gandavarapu, M. A. Azagarsamy, K. S. Anseth, Adv. Mater. 2014, 26, 2521.

[22] H. Otsuka, S. Nagano, Y. Kobashi, T. Maeda, A. Takahara, Chem. Commun. 2010, 46, 1150.
[23] Y. Amamoto, H. Otsuka, A. Takahara, K. Matyjaszewski, Adv. Mater. 2012, 24, 3975

[24] C. W. Bookwalter, D. L. Zoller, P. L. Ross, M. V. Johnston, J. Am. Soc. Mass Spectrom. 1995, 6, 872.

[25] W. Feng, L. Li, E. Ueda, J. Li, S. Heißler, A. Welle, O. Trapp, P. A. Levkin, Adv. Mater. Interfaces 2014, 1, 1400269.

[26] L. Li, W. Feng, A. Welle, P. A. Levkin, Angew. Chem., Int. Ed. 2016 55,13765 .

[27] J. Escorihuela, M. J. Bañuls, R. Puchades, Á. Maquieira, Bioconjugate Chem. 2012, 23, 2121.

[28] L. Wang, L. Li, X. Wang, D. Huang, F. Yang, H. Shen, Z. Li, D. Wu, Polym. Chem. 2016, 7, 1429.

[29] M. Kirihara, Y. Asai, S. Ogawa, T. Noguchi, A. Hatano, Y. Hirai, Synthesis 2007, 2007, 3286.

[30] L. Li, J. Li, X. Du, A. Welle, M. Grunze, O. Trapp, P. A. Levkin, Angew. Chem., Int. Ed. 2014, 53, 3835. 


\section{Repository KITopen}

Dies ist ein Postprint/begutachtetes Manuskript.

Empfohlene Zitierung:

Du, X.; Wang, M.; Welle, A.; Behboodi-Sadabad, F.; Wang, Y.; Levkin, P. A.; Gu, Z. Reparable Superhydrophobic Surface with Hidden Reactivity, Its Photofunctionalization and Photopatterning.

2018. Advanced functional materials, 28.

doi: $10.554 / I R / 1000089784$

Zitierung der Originalveröffentlichung:

Du, X.; Wang, M.; Welle, A.; Behboodi-Sadabad, F.; Wang, Y.; Levkin, P. A.; Gu, Z.

Reparable Superhydrophobic Surface with Hidden Reactivity, Its Photofunctionalization and Photopatterning.

2018. Advanced functional materials, 28 (41), Art.-Nr.: 1803765.

doi:10.1002/adfm.201803765 